\title{
GCU
}

Glasgow Caledonian

University

University for the Common Good

\section{Vehicular multitier gateway selection algorithm for heterogeneous VANET architectures}

Ansari, Shuja; Boutaleb, Tuleen; Sinanovic, Sinan; Gamio, Carlos; Krikidis, loannis

Published in:

2017 Advances in Wireless and Optical Communications (RTUWO)

DOI:

10.1109/RTUWO.2017.8228530

Publication date:

2017

Document Version

Author accepted manuscript

Link to publication in ResearchOnline

Citation for published version (Harvard):

Ansari, S, Boutaleb, T, Sinanovic, S, Gamio, C \& Krikidis, I 2017, Vehicular multitier gateway selection algorithm for heterogeneous VANET architectures. in 2017 Advances in Wireless and Optical Communications (RTUWO). IEEE, pp. 180-185. https://doi.org/10.1109/RTUWO.2017.8228530

\section{General rights}

Copyright and moral rights for the publications made accessible in the public portal are retained by the authors and/or other copyright owners and it is a condition of accessing publications that users recognise and abide by the legal requirements associated with these rights.

Take down policy

If you believe that this document breaches copyright please view our takedown policy at https://edshare.gcu.ac.uk/id/eprint/5179 for details of how to contact us. 


\title{
Vehicular Multitier Gateway Selection Algorithm for Heterogeneous VANET Architectures
}

\author{
Shuja Ansari ${ }^{1}$, Tuleen Boutaleb ${ }^{1}$, Sinan Sinanovic ${ }^{1}$, Carlos Gamio $^{1}$ and Ioannis Krikidis ${ }^{2}$ \\ ${ }^{1}$ School of Engineering and Built Environment, Glasgow Caledonian University, Glasgow, Scotland UK \\ Email: Shuja.Ansari, T.Boutaleb, Sinan.Sinanovic, Carlos.Gamio@gcu.ac.uk \\ ${ }^{2}$ Department of Electrical and Computer Engineering, Faculty of Engineering, University of Cyprus \\ Email: krikidis@ucy.ac.cy
}

\begin{abstract}
Cooperative intelligent transportation systems have become an active topic with the introduction of smart communications between vehicles, increasing driver safety, traffic efficiency and ultimately paving way for autonomous vehicles. These vehicular communications have stringent transmission requirements. Among various proposed communication protocols, use of heterogeneous networks, combining long term evolution (LTE) with dedicated short range communications (DSRC), have shown promising results. This paper proposes an LTE gateway selection procedure that enables multitier hybrid architecture. The proposed multitier heterogeneous adaptive VANET (MHAV) framework consists of two tiers, the high tier consist of authority owned vehicles or public transport operators such as buses, lorries and taxis, while low tier consist of privately owned vehicles. Having an authority owned gateway addresses the security and privacy concern raised by private car owners on sharing their information with other cars. Results from implementation of our proposed algorithm using extensive system-level simulations showed an increase in coverage area for DSRC while minimizing the number of gateway switches made by $30-35 \%$ in comparison with previously proposed multitier registration techniques. Traffic on LTE network in our simulations is also reduced by $50 \%$.
\end{abstract}

\section{INTRODUCTION}

Cooperative intelligent transportation systems (C-ITS) enable different forms of communications, such as vehicle-tovehicle (V2V), vehicle-to-infrastructure (V2I) and vehicle-topedestrian (V2P). In order to provide V2I communications, an entirely new infrastructure comprising base stations along road side are required. Installation of such an infrastructure will incur extra costs. Considering this economical issue, a number of studies have proposed the use of already installed cellular infrastructure [1]-[3]. With the presence of evolved universal mobile telecommunications service terrestrial radio access networks (E-UTRAN, referred as Long Term Evolution (LTE)), service providers and mobile network operators (MNO) have achieved high data rates with lower latencies. A number of LTE performance evaluations for the feasibility of use with vehicular ad hoc networks (VANETs) have suggested a reasonable suitability, however, without any centralization; VANETs can pose enormous network capacity issues on the cellular network [4]. With the global mobile data traffic increasing sevenfold between 2016 and 2021 [5], it can be argued whether the present network can accommodate VANETs or not, since a slight delay in message delivery, especially for autonomous vehicles, can be disastrous.

For the purpose of VANET centralization on LTE, group formation, multicast/broadcast management system (MBMS) and device-to-device (D2D) communications have been proposed [4], [6], [7]. Group formation also known as clustering has shown promising performance. However, according to [8],
$35 \%$ of road users are concerned about privacy in regards to sharing their information with other road users. At the same time, clustering relies on relaying transmissions which can pose a privacy and security issue [9]. MBMS functionality also proved to be reliable for message dissemination, although being part of 3GPP specifications, MBMS is not widely implemented by MNOs [10]. Similarly, D2D communications also referred to as LTE direct communications, using full duplex radios in order to enable vehicles to receive and transmit at the same time, have shown reduction in the use of LTE uplink resources, increasing network capacity. However, D2D for VANETs exhibits an increase in interference [7] and similar to MBMS, is not currently implemented by MNOs.

In terms of using just dedicated short range communications (DSRC) for vehicular communications, less latency is experienced as compared to LTE. However, successful message delivery in dense urban and sub-urban scenarios is not evident. For the centralization of DSRC, again there are some proposed techniques and frameworks. Among these, clustering [11] and various routing protocols [1] are some of the promising DSRC techniques. However, again with clustering or direct vehicular communication arises the concern of privacy and security.

Due to the frequent and fixed routes of public transit, studies have suggested the use of buses as mobile gateways (MG) instead of fixed road-side units (RSUs) [12]-[14]. Many advantages such as their tall structure exhibiting higher transmission range, covering most parts of urban areas, no requirement of privacy mechanisms and avoiding the cost of installing a new infrastructure, contends public buses as a good substitute for fixed base stations. Kitani et al. [13] are the first researchers who proposed using public buses for message ferrying in VANETs.

Jiang in [12] proposed BUS-VANET that integrated vehicular network with the traffic infrastructure. In their proposed framework, buses are complementing the existing ITS infrastructure. With their performance evaluation they conclude that having buses providing the same functionality as RSUs, can help offload V2I communications and decrease the number of RSUs required. The architecture implemented through simulations in downtown Minneapolis, where the bus routes are in straight lines lacked communication integration and suggested installing RSUs for ensuring service coverage.

Furthermore, authors in [14] elaborate on data aggregation technique where the buses collect beacons and service requests from the vehicles, sending them to the cloud using cellular networks. Then the cloud disseminates these beacons back to the vehicles via the same route through buses. In their study, they only present a use case where no performance is evaluated 




Fig. 1. Multitier Heterogeneous Adaptive VANET Framework showing HTNs and LTNs with all possible scenarios.

and there is no mentioning of any fall back mechanism. Li in [15], propose integrating LTE D2D communications with the existing DSRC network, enabling high tier vehicles such as taxis and public buses to form a backbone network. Most of their work is based on predicting the behavior of all the vehicles using fuzzy score logic and then routing the messages accordingly. Again there is no fall back mechanism in the absence of high tier vehicle and private cars only had DSRC capabilities.

In the same pursuit for message dissemination via public buses, Liu [16] proposes a comprehensive cloud assisted message downlink dissemination scheme. The concept presented is similar to the one proposed in [14] with a comprehensive implementation procedure. Authors have outlined how the gateway is selected, using a two-step process. The first step is for the gateway to register itself with the cloud and then the second is when a vehicle registers with the gateway. In their proposed scheme, the cloud does most of the work in the form of delegating message forwarding in a predefined targeted area. Furthermore, they assume that only the buses would have the integration of both LTE and DSRC interface, the rest of the vehicles would just use DSRC. The drawback for this framework, due to the absence of LTE interface in vehicles, is the lack of internet connectivity for ITS applications other than safety.

In the light of these previous works, we propose a framework and a gateway selection algorithm that fully integrates LTE and DSRC in order to achieve a robust system for multiple ITS applications. Contributions of this paper include:

- A multitier LTE/DSRC integrated vehicular network architecture incorporating authority and operator owned vehicles termed as multitier heterogeneous adaptive VANET (MHAV).

- High tier gateway selection algorithm for the proposed multitier heterogeneous VANET architecture.

The remainder of this paper is organized as follows: Section II describes the proposed multitier heterogenous framework, and Section III elaborates on the system model followed by simulation results in Section IV. Conclusions and future work are then discussed within Section V.

\section{Multitier Heterogeneous Adaptive VANETs}

The proposed multitier heterogeneous adaptive VANET (MHAV) framework incorporates high tier nodes (HTN) and low tier nodes (LTN). HTNs are the authority owned vehicles such as public buses, taxies, council lorries, etc. while LTNs comprise all the other private vehicles. Both HTNs and LTNs are assumed to be equipped with LTE and DSRC interface, integrated with the help of a control device [17].

Data delivery in the proposed framework is carried out with the corporation of HTNs, traffic control center (TCC) and vehicular safety application (VSA) server. The TCC and VSA are situated at the core of LTE network and are also accessible via internet. All the LTNs get registered with HTNs, which then enables V2I communications. If an HTN is not available, LTN falls back to using LTE network. HTNs consistently communicate with the LTE network, updating the traffic conditions and their registered LTNs table with the TCC and VSA. HTNs broadcast beacons every second consisting of their location, velocity and ID using DSRC. LTNs receiving these broadcasts run our proposed registration algorithm in order to register with the most suitable HTN. Once the LTN is registered, all $\mathrm{V} 2 \mathrm{~V}$ communications are carried out via the registered HTN, acting as a message relay. The basic architecture of MHAV framework is shown in Figure 1.

Since all the traffic related information is updated in the TCC, LTNs not registered with HTNs can also access this information via LTE. In regards to safety applications, we suggest the use of a differentiated quality of service (QoS) mechanism known as safety application identifier (SAI) proposed in [18] and implemented via the VSA server. In the next subsection, we explain the proposed HTN selection algorithm which is implemented at the LTN. Furthermore, MHAV framework has a number of other benefits such as no clustering, efficient adaptation, more applicability, etc., a brief comparison is shown in Table I.

\section{A. HTN Selection Algorithm}

By having HTNs with integrated DSRC and LTE, we design a system where the HTNs act as gateways. In order to select an HTN, LTNs run our proposed algorithm every time a broadcast beacon is received. Similar to the scheme proposed in [12], each HTN maintains a registration table recording the LTNs currently registered with them. These tables are constantly reported and updated with TCC over the LTE network. In order to have a robust network, especially with such a mobile topology, determining which HTN to select for registration is an important issue when LTNs can receive multiple broadcast beacons from a number of HTNs.

When an LTN receives a broadcast beacon from HTN, this node is placed in the candidate registration set $(S)$. Using the information in the broadcasted beacon, LTN calculates the connection delivery delay $(T)$ for every HTN in the candidate registration set. This delay is calculated using HTN's transmission range $(R)$ which is predefined, distance between the HTN and LTN $(d)$ and relative velocity $\left(v_{L T N}-v_{H T N}\right)$. Negative value of this delay means that the HTN is moving in the opposite direction to the LTN, therefore if $T$ is negative the HTN is placed in the discard set. Out of all the HTNs residing in $S$, the one with the highest $T$ is selected for registration. Once LTN has registered with the HTN, it stays connected with it until the distance between LTN and HTN remains below the predefined transmission range. This is elaborated in Algorithm 1.

Authors in [12] had a similar concept, where they set a threshold delivery delay. Setting this threshold and enabling multi hop approach avoids ping pong effect but results in high delays. To tackle this ping pong problem in our evaluations, we preset the $R$ and force the LTN to stay connected with 
TABLE I

Related Work on Multitier Heterogeneous VANETs

\begin{tabular}{|c|c|c|c|c|c|c|c|c|c|c|}
\hline Algorithm & $\begin{array}{l}\text { Tier } \\
\text { Nodes }\end{array}$ & $\begin{array}{l}\text { LTE- } \\
\text { DSRC }\end{array}$ & $\begin{array}{l}\text { LTE } \\
\text { in all } \\
\text { nodes }\end{array}$ & Clusters & Fall-back & $\begin{array}{l}\text { Simulation } \\
\text { Area }\end{array}$ & Application & $\begin{array}{l}\text { Selection Cri- } \\
\text { teria }\end{array}$ & $\begin{array}{l}\text { Performance } \\
\text { Metrics }\end{array}$ & Ref \\
\hline $\begin{array}{l}\text { BUS- } \\
\text { VANET } \\
\text { LRTA }\end{array}$ & $\begin{array}{l}\text { Buses/ } \\
\text { Private }\end{array}$ & No & No & Yes & None & $\begin{array}{l}\text { Downtown } \\
\text { Minneapolis, } \\
\text { USA }\end{array}$ & Safety & $\begin{array}{l}\text { Maximum de- } \\
\text { livery delay }\end{array}$ & $\begin{array}{l}\text { Delivery Delay, } \\
\text { Packet Loss }\end{array}$ & [12] \\
\hline TPHVN & $\begin{array}{l}\text { Buses, } \\
\text { Taxis/ } \\
\text { Private }\end{array}$ & Yes & No & Yes & Clustering & $\begin{array}{l}\text { Tianjin } \\
\text { Nankai, } \\
\text { China }\end{array}$ & Safety & $\begin{array}{lr}\text { Fuzzy } & \text { score of } \\
\text { vehicle } & \text { type } \\
\text { and } & \text { traffic } \\
\text { speed }\end{array}$ & $\begin{array}{l}\text { Delivery Ratio, } \\
\text { Delivery Delay }\end{array}$ & [15] \\
\hline CMDS & $\begin{array}{l}\text { Buses/ } \\
\text { Private }\end{array}$ & Yes & No & Yes & Clustering & $\begin{array}{l}\text { Highway/ } \\
\text { Grid Model }\end{array}$ & Safety & $\begin{array}{l}\text { Cloud decides } \\
\text { using transmis- } \\
\text { sion range }\end{array}$ & $\begin{array}{l}\text { Dissemination } \\
\text { Delay }\end{array}$ & [16] \\
\hline $\begin{array}{l}\text { MHAV } \\
\text { (Pro- } \\
\text { posed) }\end{array}$ & $\begin{array}{l}\text { Authority } \\
\text { owned } \\
\text { buses, } \\
\text { taxis, } \\
\text { lorries/ } \\
\text { private }\end{array}$ & Yes & Yes & No & $\begin{array}{ll}\text { LTE } & \text { SAI } \\
{[18]} & \end{array}$ & $\begin{array}{l}\text { Glasgow } \\
\text { City Centre, } \\
\text { UK }\end{array}$ & $\begin{array}{l}\text { Safety, Traf- } \\
\text { fic efficiency } \\
\text { and Infotain- } \\
\text { ment }\end{array}$ & $\begin{array}{l}\text { Relative } \\
\text { velocity, } \\
\text { location and } \\
\text { transmission } \\
\text { range }\end{array}$ & $\begin{array}{l}\text { Delivery Delay, } \\
\text { Packet Loss, } \\
\text { LTE Network } \\
\text { quality }\end{array}$ & - \\
\hline
\end{tabular}

the registered HTN until it moves out of the transmission range. To select an optimum $R$, we tested our system under varying values of $R$. The results showed a trade off between number of registration switches and DSRC coverage area. The results also predict that for suburban, rural or highway scenarios this range $R$ will vary accordingly. More details regarding the selection of this range parameter is discussed in Section IV. Next we discuss the system model adopted for our performance evaluations.

\section{SySTEM MODEL}

The network modeled is a $2 \times 2 \mathrm{~km}^{2}$ area of Glasgow city center with varying density of vehicles evaluating both rush hours when there is high presence of HTNs and less busy hours with lesser HTNs available. Both LTNs and HTNs are assumed to be equipped with FDD LTE transceivers with $20 \mathrm{MHz}$ bandwidth, uplink carrier frequency $1715 \mathrm{MHz}$ and downlink carrier frequency $2115 \mathrm{MHz}$ (band 4) [19, Table 5.51] integrated with IEEE $802.11 \mathrm{p}$ compliant DSRC interface operating at $5.9 \mathrm{GHz}$ with $10 \mathrm{MHz}$ bandwidth [20]. These nodes are assumed to be moving in urban model created using routes mobility model [21]. Fig. 2 illustrates the service area modeled in ns-3 [22]. Nodes move at an urban average speed matched to the 3GPP extended vehicular A (EVA) model radio environment designed using MATLAB [23]. Simulation parameters used are given in Table II.

Furthermore for HTNs, predefined bus routes are modeled with an interval of 10 minutes [24]. For the eNodeBs (eNBs), mast data for operator EE has been implemented [25]. The eNBs are connected to the mobility management entity (MME) through their S1-AP interface and to the serving gateway $(\mathrm{S}-\mathrm{GW})$ and packet data network gateway (P-GW) through their S1-U interfaces. Interconnection from the P-GW to the TCC Server and VSA server is modeled using an error free 10 Gbps point-to-point link and TCP/IP version 4 . The packet payload for HTNs is assumed to be 1500 bytes including the registration tables, locations and safety application data.

Propagation loss model employed for IEEE 802.11p is Nakagami loss model with the path loss factor $(m)$ of 4 [26] on top of Friis propagation loss model. Multiple simulation runs are undertaken in order to obtain reliable results. The results from all these simulations are then averaged using $95 \%$ confidence interval. LTN velocity is set to $30 \mathrm{mph}$ while the
HTNs are assumed to be moving at $20 \mathrm{mph}$, according to the city speed limits enforced in Glasgow city center.

\section{A. Performance Measures}

We compare our results with the previously proposed longest registration time algorithm implemented in BUSVANETs [12]. The primary performance measures used are the average number of registration switches, IEEE $802.11 p$ coverage and the packet delivery ratio $(P D R)$. The registration switches are calculated for each LTN whenever it switches from an HTN to either another HTN or to the LTE network. IEEE 802.11p coverage is the percentage of LTNs registered with HTN. Furthermore, the $P D R$ is the number of packets successfully delivered to the LTNs after they have registered with the respective HTN.

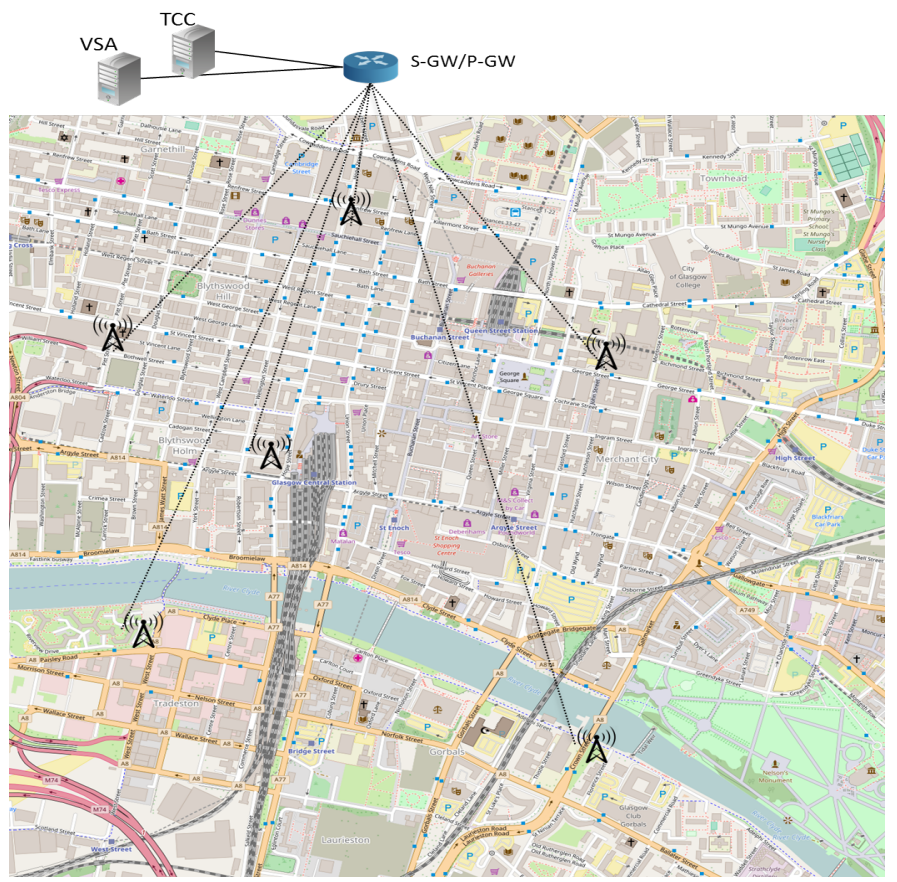

Fig. 2. $2 \times 2 \mathrm{~km}^{2}$ area of Glasgow city center covered by 6 sites with 3 cells/site. 


\section{Simulation Results}

Vehicular networks have a fast changing topology due to their mobile nature. In MHAV framework, LTNs carry out $\mathrm{V} 2 \mathrm{~V}$ communications via HTNs. Therefore, the amount of registration switching between HTNs is required to be low for the network to be robust and reliable. For the purpose of evaluation, we tested our system while varying the transmission range, in order to select the optimum algorithm parameter. Once a suitable transmission range is selected, we compare our algorithm with the registration scheme presented in [12] referred as longest registration time algorithm (LRTA), concluding with the overall outcomes of our study.



TABLE II

SimUlation PARAMETERS

\begin{tabular}{l|l} 
Parameter & Value \\
\hline \hline Simulation time & 300 seconds. \\
Road model & $2 \times 2 \mathrm{~km}^{2}$ Glasgow City Center \\
Number of LTNs & $50,100,150$. \\
Number of HTNs & $5,10,15$. \\
Average speed & $20 \mathrm{mph}(\mathrm{HTN}), 30 \mathrm{mph}(\mathrm{LTN})$. \\
DSRC & \\
Access Technology & IEEE WAVE 1609 and $802.11 \mathrm{p}$. \\
Propagation model & Nakagami and Friis Models. \\
Operating Frequency & $5.9 \mathrm{GHz}$. \\
Data Rate & $6 \mathrm{Mbps}$. \\
Transmission Power & $25 \mathrm{dBm}$. \\
Antenna & Omnidirectional. \\
Channel Bandwidth & $10 \mathrm{MHz}$. \\
Noise Figure & $7 \mathrm{~dB}$. \\
CCA threshold & $-86 \mathrm{dBm}$. \\
LTE & \\
Network & 6 sites with 3 cells/site, $1000 \mathrm{~m} \mathrm{ISD.}$ \\
Transmission power & eNB: $40 \mathrm{dBm}$, UE: $23 \mathrm{dBm}$. \\
Carrier frequency DL/UL & $2115 \mathrm{MHz} / 1715 \mathrm{MHz}$. \\
Channel bandwidth & $20 \mathrm{MHz}(100 \mathrm{RBs})$ \\
Noise Figure & eNB: $5 \mathrm{~dB}, \mathrm{UE}: 9 \mathrm{~dB}$. \\
UE antenna model & Isotropic $(0 \mathrm{dBi})$. \\
eNB antenna model & $15 \mathrm{~dB}$ Cosine model, $65^{\circ} \mathrm{HPBW}$. \\
Scheduling algorithm & Proportional Fair. \\
Handover algorithm & A2A $4 \mathrm{RSRQ}$, RSRQ threshold $-5 \mathrm{~dB}$, \\
& and NeighbourCellOffset=2 $(1 \mathrm{~dB})$. \\
Path loss model & LogDistance $(\alpha=3)$ and \\
& $3 \mathrm{GPP}$ Extended Vehicular A model. \\
\hline
\end{tabular}

\section{A. Selection of Transmission Range for Urban Scenario}

As mentioned in Section II-A, mechanism reducing rapid topology change increases robustness, however, another vital requirement is the reliability. Since vehicular network's safety applications require successful message delivery, the tradeoff between registration switches and message delivery rate is evaluated. At the same time, we assess the amount of traffic that would be offloaded from the LTE network to the proposed IEEE 802.11 p network, reducing the capacity usage of VANETs on LTE network.

Fig. 3 shows a 3D plot with PDR on the horizontal axis while the average number of switches and IEEE 802.11p coverage on $x$ and $z$ axis respectively. Two scenarios are evaluated where the number of HTNs is varied from 10 to 15. We have carried out our tests with the transmission ranges of 100,200 and $300 \mathrm{~m}$. For $R=300 \mathrm{~m}$, system with 15 HTNs had DSRC covering above $80 \%$ of LTNs with the least average registration switching of 2.1, however, the PDR was found to be close to $60 \%$. With the same transmission range, having 10 HTNs in the scenarios, almost $65 \%$ of the LTNs were observed to be using DSRC for communication, while the PDR further dropped to below 50\%. The low value of PDR shows that while the switching is minimal and the DSRC coverage is optimal, quite a large amount of packets are dropped. This is due to the fading and shadowing effect in an urban environment, due to the presence of buildings.

Next we changed $R$ to $200 \mathrm{~m}$. As expected, the DSRC coverage decreased while the number of registration switching increased, however, the PDR increased by about $15 \%$ in both scenarios having 15 and 10 HTNs. Furthermore, we calculated that on average a block in Glasgow city center is slightly less than $100 \mathrm{~m}$ which would avoid fading and shadowing effects caused due to the presence of buildings. Therefore, we then used $R=100 \mathrm{~m}$ and observed the PDR going above $85 \%$ for both the scenarios. The trade-off, as predicted, is with 




Fig. 3. Packet Delivery Rate vs IEEE 802.11 p coverage and Number of switches for 10 and 15 HTNs with 100 LTNs while changing the transmission range from $100 \mathrm{~m}$ to $300 \mathrm{~m}$.

the DSRC coverage and the average number of registration switches. For 15 HTNs at $R=100 \mathrm{~m}$, about $68 \%$ of LTNs are covered by DSRC while the average switching remains at about 2.6, exhibiting a PDR of above $90 \%$. Whereas for 10 HTNs, the switching goes above 3 while DSRC coverage is above $50 \%$ with a PDR of $85 \%$.

From a literature study carried out in [27], traditional VANETs have a PDR of between $60-80 \%$. However, the standards do not specify any acceptable packet delivery ratio. It can hence be concluded that in an urban environment such as Glasgow city center, transmission range of $100 \mathrm{~m}$ shows the most reliability (above 85\%) while trading off with the number of switching and DSRC coverage. With this PDR, the coverage is still above $50 \%$ showing an offload of more than half the traffic from LTE network.

\section{B. Comparison of MHAV Registration Scheme with LRTA}

Next we compare our proposed algorithm with the previously proposed [12] LRTA for bus VANETs. Fig. 4 shows the average number of registration switches that occurred during the $300 \mathrm{sec}$ of simulation. The average is taken for each LTN and then $95 \%$ confidence interval is calculated over multiple simulation runs. It can be seen that the number of switches decrease by about $30 \%$ to $35 \%$ when our proposed registration scheme is in place. The reason behind this decrease is the selection of threshold parameters. For LRTA, the threshold

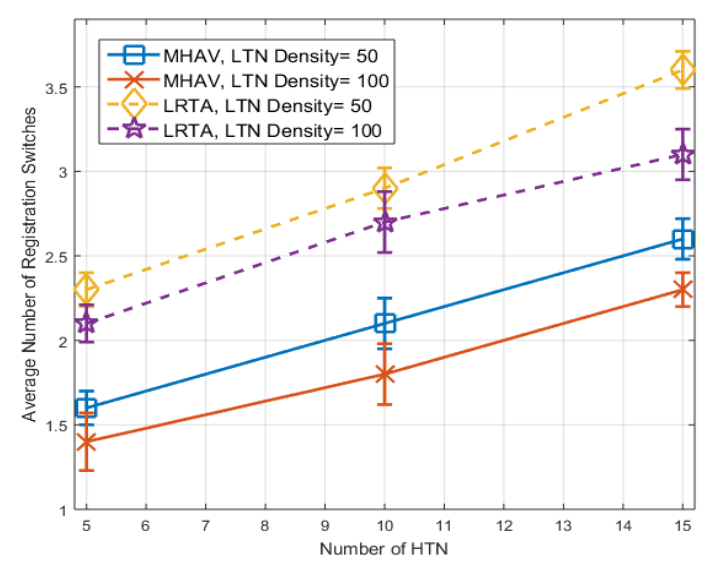

Fig. 4. Comparison of MHAV with LRTA on the average number of registration switches. chosen is the delivery delay, i.e., whenever this delay falls below a certain threshold, the LTN registers with the HTN and stays connected until this value crosses the threshold. For a fast vehicle in a radio environment, this delay can arise from various sources and multiple HTNs can have similar delays, specially when multiple HTNs are present at the same location. Our selection of transmission range instead of delivery delay shows higher robustness, since the LTN tends to stay registered with one HTN till the time it is present in the predefined transmission range.

\section{CONClusion And Future Work}

This paper proposes a multitier heterogeneous adaptive VANET framework and a gateway selection algorithm. MHAV architecture consists of HTN and LTN. All the vehicles are assumed to have LTE and DSRC capabilities while LTNs register with HTNs to enable V2I and V2V communications over DSRC while the HTNs connect to the LTE network in order to provide infrastructure communications to its registered LTNs. We also propose to have a fall-back to LTE SAI in the case where there's no HTN present for registration. Simulations are carried out in Glasgow city center, a dense urban environment, in order to evaluate our proposed algorithm. Results show that the proposed scheme outperforms the traditional BUS VANET registration technique by $30-35 \%$ in terms of switching while offloading more than half of the vehicular traffic from cellular networks and maintaining a PDR above $85 \%$. Having authority owned gateways tend to make the network more secure and addresses the privacy issue raised by many private car owners.

In the future, we plan to implement a message dissemination scheme for the proposed framework, expanding it to meet requirements for all the vehicular applications. We will also evaluate other scenarios such as highways and suburban areas where the transmission range parameter in our algorithm is speculated to be significant. LTE has been a promising candidate for vehicular networks. However with the current growth in cellular users, catering vehicular networks would require much more capacity. We plan to implement techniques that would integrate LTE and DSRC while minimizing the impact of capacity issue.

\section{REFERENCES}

[1] S. Al-Sultan, M. M. Al-Doori, A. H. Al-Bayatti, and H. Zedan, "A comprehensive survey on vehicular ad hoc network," Journal of network and computer applications, vol. 37, pp. 380-392, 2014.

[2] K. Tanuja, T. Sushma, M. Bharathi, and K. Arun, "A survey on VANET technologies," International Journal of Computer Applications, vol. 121, no. $18,2015$.

[3] A. Fasbender, M. Gerdes, and S. Smets, "Cellular networking technologies in its solutions: opportunities and challenges." Springer Berlin Heidelberg, 2012, pp. 1-13. [Online]. Available: http://dx.doi.org/10.1007/978-3-642-29667-3_1

[4] G. Remy, S.-M. Senouci, F. Jan, and Y. Gourhant, "LTE4v2x: LTE for a Centralized VANET Organization," in IEEE Global Telecommunications Conference. Houston, TX, USA: IEEE, Dec. 2011, pp. 1-6.

[5] Cisco, "Cisco Visual Networking Index : Global Mobile Data Traffic Forecast Update , 2016 - 2021,” Tech. Rep., 2017.

[6] Y. Yang, P. Wang, C. Wang, and F. Liu, "An eMBMS based congestion control scheme in cellular-VANET heterogeneous networks," in 17th International IEEE Conference on Intelligent Transportation Systems (ITSC), Oct. 2014, pp. 1-5.

[7] A. Bazzi, B. M. Masini, and A. Zanella, "Performance Analysis of V2V Beaconing Using LTE in Direct Mode With Full Duplex Radios," IEEE Wireless Communications Letters, vol. 4, no. 6, pp. 685-688, Dec 2015.

[8] B. Schoettle and M. Sivak, "A survey of public opinion about autonomous and self-driving vehicles in the us, the uk, and australia," 2014. 
[9] S. Taha and X. Shen, "A physical-layer location privacy-preserving scheme for mobile public hotspots in nemo-based vanets," IEEE Transactions on Intelligent Transportation Systems, vol. 14, no. 4, pp. 16651680, Dec 2013.

[10] Public warning system (PWS) requirements (release 13), 3GPP TS 22.268 V13.0.0, Dec. 2015.

[11] S. Ucar, S. C. Ergen, and O. Ozkasap, "Multi-hop cluster based IEEE $802.11 \mathrm{p}$ and LTE hybrid architecture for VANET safety message dissemination," IEEE Transactions on Vehicular Technology, vol. PP, no. 99, pp. 1-1, 2015.

[12] X. Jiang and D. H. C. Du, "Bus-vanet: A bus vehicular network integrated with traffic infrastructure," IEEE Intelligent Transportation Systems Magazine, vol. 7, no. 2, pp. 47-57, Summer 2015.

[13] T. Kitani, T. Shinkawa, N. Shibata, K. Yasumoto, M. Ito, and T. Higashino, "Efficient vanet-based traffic information sharing using buses on regular routes," in VTC Spring 2008 - IEEE Vehicular Technology Conference, May 2008, pp. 3031-3036.

[14] R. Hussain, F. Abbas, J. Son, S. Kim, and H. Oh, "Using public buses as mobile gateways in vehicular clouds," in 2014 IEEE International Conference on Consumer Electronics (ICCE), Jan 2014, pp. 175-176.

[15] G. Li, M. Ma, C. Liu, and Y. Shu, "Routing in taxi and public transport based heterogeneous vehicular networks," in 2016 IEEE Region 10 Conference (TENCON), Nov 2016, pp. 1863-1866.

[16] B. Liu, D. Jia, J. Wang, K. Lu, and L. Wu, "Cloud-assisted safety message dissemination in vanet cellular heterogeneous wireless network," IEEE Systems Journal, vol. 11, no. 1, pp. 128-139, March 2017.

[17] D. Serrano, O. Klemp, H. Lim, and M. Strassberger, "Control of vehicle communication using two protocols," Oct. 18 2016, US Patent 9,473,579. [Online]. Available: https://encrypted.google.com/patents/US9473579

[18] S. Ansari, T. Boutaleb, C. Gamio, S. Sinanovic, I. Krikidis, and M. Sánchez, "Vehicular Safety Application Identifier algorithm for LTE VANET server," in 2016 8th International Congress on Ultra Modern Telecommunications and Control Systems and Workshops (ICUMT), Oct 2016, pp. 37-42.

[19] E-UTRA Base Station (BS) radio transmission and reception (Release 12), 3GPP TS 36.104 V12.10.0, Jan. 2016.

[20] IEEE standard for wireless access in vehicular environments (WAVE) networking services, IEEE Std. Std 1609. $3^{T M}-2010$, Dec. 2010.

[21] T. Cerqueira and M. Albano, "Routesmobilitymodel: Easy realistic mobility simulation using external information services," in Proceedings of the 2015 Workshop on Ns-3, ser. WNS3 '15. New York, NY, USA: ACM, 2015, pp. 40-46. [Online]. Available: http://doi.acm.org/10.1145/2756509.2756515

[22] Model library release ns-3.2, Ns-3 network simulator, 2015. [Online] Available: https://www.nsnam.org/docs/models/html/lte-design.html

[23] G. T. . V8.2.0, Evolved Universal Terrestrial Radio Access (E-UTRA), Base Station (BS) radio transmission and reception (Release 8). 3GPP, May 2008.

[24] "Timetables - greater glasgow." [Online]. Available: https://www.firstgroup.com/greater-glasgow/plan-journey/timetables/

[25] "Cellular coverage and tower map for ee network in glasgow." [Online] Available: https://www.cellmapper.net/

[26] J. Goldhirsh and W. J. Vogel, "Handbook of propagation effects for vehicular and personal mobile satellite systems," NASA Reference Publication, vol. 1274, pp. 40-67, 1998.

[27] W. Sun, T. Fu, Y. Su, F. Xia, and J. Ma, "ODAM-C: an improved algorithm for vehicle ad hoc network," in International Conference on Internet of Things, and 4th International Conference on Cyber, Physical and Social Computing (iThings/CPSCom), Oct 2011, pp. 152-156. 